\title{
Validation of the new filters configuration for the RPC gas systems at LHC experiments
}

\author{
Beatrice Mandelli ${ }^{1}$ \\ CERN, University of Oslo \\ CH-1211 Geneva, Switzerland \\ E-mail: beatrice.mandelli@cern.ch

\section{Capeans, R. Guida, F. Hahn, S. Haider} \\ CERN \\ CH-1211 Geneva, Switzerland \\ E-mail:mar.capeans@cern.ch, roberto.guida@cern.ch, \\ ferdinand.hahn@cern.ch, stefan.haider@cern.ch
}

\begin{abstract}
Resistive Plate Chambers (RPCs) are widely employed as muon trigger systems at the Large Hadron Collider (LHC) experiments. Their large detector volume and the use of a relatively expensive gas mixture make a closed-loop gas circulation unavoidable. The return gas of RPCs operated in conditions similar to the experimental background foreseen at LHC contains large amount of impurities potentially dangerous for long-term operation. Several gas-cleaning agents, characterized during the past years, are currently in use. New test allowed understanding of the properties and performance of a large number of purifiers. On that basis, an optimal combination of different filters consisting of Molecular Sieve (MS) $5 \AA$ and $4 \AA$, and a $\mathrm{Cu}$ catalyst R11 has been chosen and validated irradiating a set of RPCs at the CERN Gamma Irradiation Facility (GIF) for several years. A very important feature of this new configuration is the increase of the cycle duration for each purifier, which results in better system stability and, if needed, it would permit to increase the gas flow in the detectors during the high luminosity running periods at LHC. During the test of the new filters configuration, the detector performances were monitored in terms of current stability and Bakelite resistivity.
\end{abstract}

XI workshop on Resistive Plate Chambers and Related Detectors (RPC2012)

INFN-Laboratori Nazionali di Frascati, Italy

February 5-10, 2012

\footnotetext{
$1 \quad$ Speaker
} 


\section{Introduction}

Resistive Plate Chambers (RPCs) are widely employed for the muon trigger systems at the Large Hadron Collider (LHC) thanks to their fast time resolution ( $\sim 1 \mathrm{~ns})$, suitable space resolution $(\sim 1 \mathrm{~cm})$ and low production cost $\left(\sim 100 € / \mathrm{m}^{2}\right)$. Most RPC systems at LHC are operated in a non-flammable gas mixture of $\mathrm{C}_{2} \mathrm{H}_{2} \mathrm{~F}_{4}-\mathrm{iC}_{4} \mathrm{H}_{10}-\mathrm{SF}_{6}-\mathrm{H}_{2} \mathrm{O}$, which is relative expensive $\left(\sim 60 € / \mathrm{m}^{3}\right)$. Based on economical grounds and related to the large system size ( 4000 $\mathrm{m}^{2}$ ), RPCs are operated in re-circulating gas systems (90-95\% closed loop circulation).

Several studies indicate a clear correlation between RPC performance and the quality of the gas mixture [1]. The current drawn by the chambers can rapidly rise if the amount of pollutants in the mixture increases, be due to poor gas quality at the source (e.g. polluted Freon bottles), the accumulation of impurities due to malfunctioning or saturated gas purifiers, leaks in the gas system, etc. Furthermore in the high radiation environment expected at LHC many different, chemically reactive impurities are created in the RPC gas, mainly hydrocarbons, HF, $\mathrm{F}^{-}$and Freon-type molecules. They are potentially dangerous for the detector materials, the gas system and ultimately will degrade the detector performances.

This study investigates the impurities produced in heavily irradiated RPC chambers and the properties of possible absorbers to be used at LHC in order to keep the gas purity near the level of the fresh gas quality. Results of these tests have revealed an optimized configuration of filters that is now being implemented in the closed-loop gas system of the ATLAS and CMS RPC systems.

\section{Experimental set-up}

The experimental setup consists of a set of double gap Bakelite RPCs that are irradiated at the CERN Gamma Irradiation Facility [2], GIF (590 GBq ${ }^{137} \mathrm{Cs}$ source). Each detector has a surface of $120 \times 205 \mathrm{~cm}^{2}$ and a $2 \mathrm{~mm}$ wide gas gap. In the double gap layout used for this test, the two gaps are connected in series (i.e. the output of the first gap is the input of the second and the read-out strips are in between the two gaps). All gaps were part of the standard production for the LHC experiments. The detectors are connected to a versatile closed-loop gas system with several gas analysers [3]. This gas system is a small replica of the gas systems installed in the LHC experiments and furthermore purifiers can be studied in detail, at the same time that chambers behavior is regularly monitored. This system, in addition, allows the simultaneous operation of two sets of RPCs in different gas conditions: one set is operated in open-mode (i.e. the gas is exhausted to atmosphere) and it serves to monitor chamber performances in ideal conditions; the second set is connected to the closed-loop gas circuit, to test the chamber performance for a given gas quality condition. 
In the current tests, the RPCs were located at about $2 \mathrm{~m}$ from the ${ }^{137} \mathrm{Cs}$ source resulting in an average counting rate of $200 \mathrm{~Hz} / \mathrm{cm}^{2}$ over almost the whole surface, and a dose rate of $1 \mathrm{cGy} / \mathrm{h}$ (the overall acceleration factor, with respect to the normal operation in the LHC experiments at nominal luminosity, is about 30). The RPCs were placed inside a tent where temperature and relative humidity were regulated to about $20^{\circ} \mathrm{C}$ and $40 \%$ respectively.

\subsection{Gas analysis}

The gas quality is a fundamental parameter for the RPC performances. Thus, several gas analysers have been selected and are continuously used to characterize in detail the gas quality at different sampling points at the GIF and at the LHC gas systems.

Several studies have been conducted in previous tests [1], [3] concerning the fluoride ions, which are largely produced inside the gas volume during RPC operation and are very reactive impurities. These studies proved that the fluoride ions present in the gas mixture are harmful to RPCs. A systematic study has been conducted [1] using a double channel ISE station allows the simultaneous measurement of fluoride ions concentration in different detectors or different gas sampling points.

In addition to the important amount of fluoride ions measured at the exhaust of irradiated RPCs, many other molecules are produced as a result of the breaking of the main gas mixture components by means of ionization, UV photons, charge multiplication and the subsequent recombination/re-arrangement of fragments. In order to identify unknown pollutants, an Agilent 3000 microGas Chormatograph (microGC) coupled to an Agilent 5975 mass spectrometer detector (MSD) is used to detect, quantify and identify pollutants. The microGC is in fact a set of 3 independent microGC modules with own micro injector, analytical column and Thermal Conductivity Detector. The analytical columns are OV1, PoraPlotU (PPU) and MS $5 \AA$, which can separate heavy hydrocarbons (from $\mathrm{C}_{4}$ to $\mathrm{C}_{12}$ ), light hydrocarbons (from $\mathrm{C}_{1}$ to $\mathrm{C}_{4}$ ) plus $\mathrm{H}_{2} \mathrm{O}$, $\mathrm{CO}_{2}, \mathrm{CH}_{4}$ and light components plus noble gases respectively [4]. Thanks to the MSD is then possible to identify each separated compound.

\section{Long-term validation of highly irradiated RPCs in an optimized closed-loop gas system}

Based on the detailed understanding and systematic characterization of several purifiers [5], three different configurations of purifiers have been tested at the GIF set-up during the years 2009-2011. Fig. 1 shows the average integrated charge by irradiated RPCs versus time; the total accumulated charge is equivalent to about 10 years of operation in the LHC systems.. The test time is subdivided into four intervals:

1. 'Set-up validation' corresponds to the irradiation of RPCs operated in an open mode gas system. It serves to optimize and validate the GIF set-up, the RPC chambers and to characterize in detail and individually different filters exposed to exhausted gas from the heavily irradiated RPCs. 
2. The period 'Current configuration at LHC experiments' is a long-term, systematic test of the combination of purifiers currently in use in the LHC RPC systems; therefore it aims at revealing the possible limitations of the current RPC closed-loop gas systems for the future higher luminosity running.

3. The third period is a test of the so-called 'first optimized configuration', using a new combination of purifiers.

4. The last period corresponds to the so-called 'second optimized configuration', which simplifies the previous filter's combination while it improves the overall performance of the gas system.

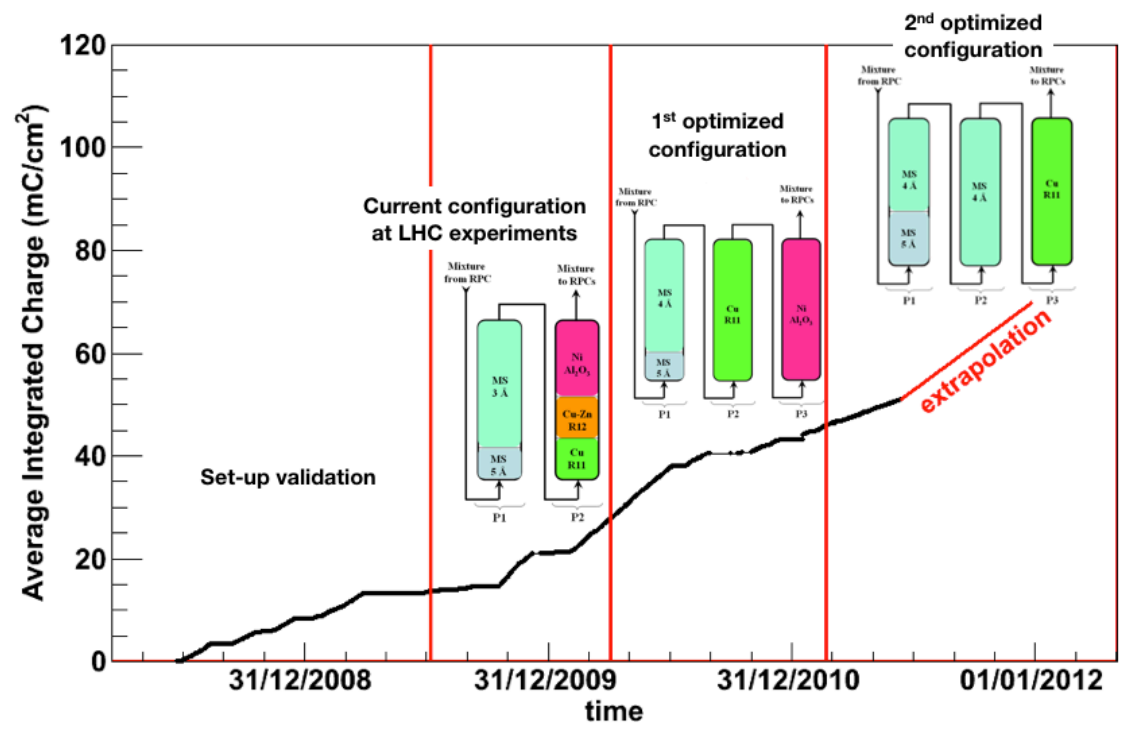

Fig. 1 Average integrated charge seen by the irradiated RPCs from the beginning of the test. Four different time periods corresponding to the test of different purifier configurations are visible. For each test period, the purifiers configurations are also visible.

\subsection{Optimization of the filters configuration}

As a result of the detailed and systematic characterization of each individual filter, it has been found that there is a combination of purifiers that would filter more efficiently some of the gas pollutants typically present in the irradiated RPC mixture, in addition to $\mathrm{H}_{2} \mathrm{O}$ and $\mathrm{O}_{2}$. The so-called 'first optimized configuration' consists of four different absorbers (Fig. 1). In comparison to the current LHC configuration:

- The MS $3 \AA$ has been replaced by $4 \AA$, which permits to reduce the conditioning time and it absorbs more efficiently water and RPC-related impurities;

- The catalyst CuZn R12 has been removed, as it does not filter any of the radiationinduced impurities. The remaining metallic catalysts double their volume (by using two individual cartridges instead of one) and so their cleaning capacity, especially for $\mathrm{O}_{2}$; 
In this configuration one disadvantage remains: the low water and Freon-like impurities absorption capacity due to the presence of only one cartridge of MS.

In order to correct this limitation, the second optimized configuration consists of three physical cartridges, two of which have an important abundance of MS $4 \AA$ (Fig. 1). The advantages with respect to the other two configurations are:

- The water absorption capacity is increased by a factor 2, and the increased amount of MS also guarantees an effective filtering of RPC-pollutants.

- The metallic catalyst $\mathrm{Ni}-\mathrm{Al}_{2} \mathrm{O}_{3}$ is abandoned because it has shown that under some conditions can pollute the gas mixture. $\mathrm{Cu}$ R11 remains as the unique $\mathrm{O}_{2}$ filter.

\subsection{RPC gas quality using optimized filter configurations}

In order to characterize in detail the impurities produced in heavily irradiated RPCs, and the filtering capacity of several filter configurations, systematic analysis of the composition of the RPC gas mixture by means of the microGC/MSD were carried out daily. Six sampling points are available in the gas system:

- Point 1 (FreshMix): fresh mixture delivered by the gas mixer;

- Point 2 (OMReturn): the gas mixture from the chambers exhaust in the open-mode gas circuit;

- Point 3 (CLReturn): the gas mixture from the chambers exhaust in the closed-loop gas circuit;

- Points 4-5-6 (AfterP1-P2-P3): the gas mixture after the first, second and third purifiers respectively.

In these six points the correct proportion of the gas mixture and the impurities concentration down-stream the detectors are calculated and monitored.

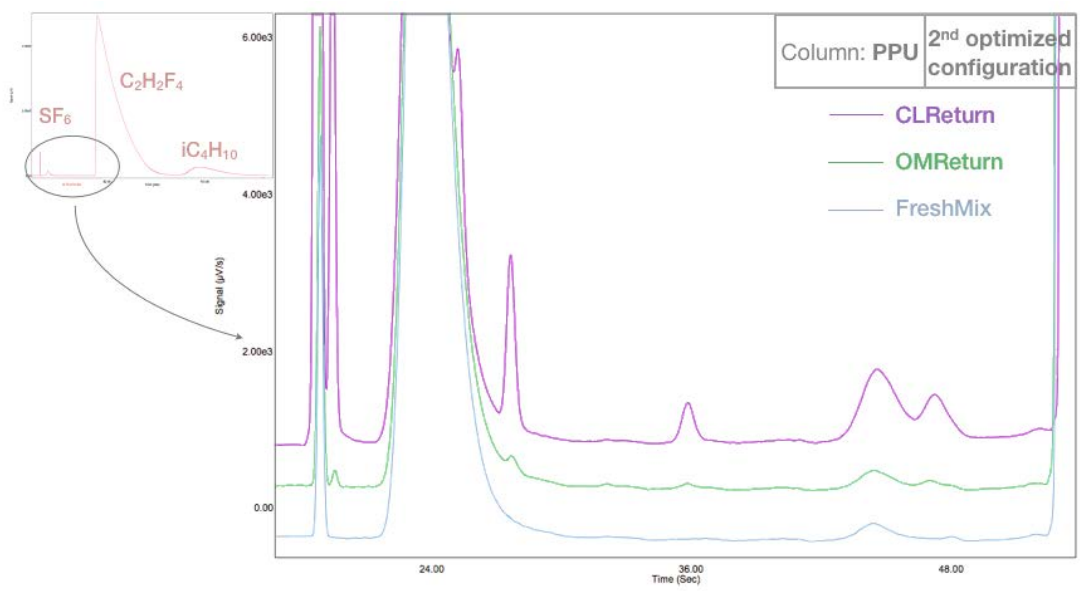

Fig. 2 Comparison between three gas chromatograms (obtained with column PPU) from the following analysis points during test of the second optimized configuration: fresh mixture gas (FreshMix), gas exiting the irradiated RPC in open mode (OMReturn) and gas mixture after the chamber in closed-loop (CLReturn) [6]. 
Fig. 2 shows a comparison of several gas chromatograms. For the gas mixture extracted after the chambers operated in closed-loop (CLReturn), impurities are clearly visible and their concentration is higher than in the gas from chambers operated in open mode. These impurities, accumulated in the closed-loop return gas mixture, can damage the RPCs and reduce their performances in time.

\subsubsection{Gas mixture humidity}

A crucial parameter for the stable operation of RPC detectors is the relative humidity inside the gap. The amount of water vapor injected into the mixture should be constant as it helps defining the electrical properties of the Bakelite electrodes. In order to control the relative humidity in the closed-loop, which should be about $40 \%$, the water in the return mixture from irradiated chambers is completely removed by means of MS purifiers. Then, the right water concentration is freshly injected in the chambers' supply line.

The concentration of $\mathrm{H}_{2} \mathrm{O}$ in the filtered gas (after purifiers 1, 2 and 3) was monitored all the long test period. In Fig. 3 it can be noticed that in the 'current LHC configuration' and in the 'first optimized configuration' there is about the same amount of water after the purifiers, between 100 and 300 ppm. The highest levels are reached when the MS purifiers are saturated, and slowly decrease to the hundred-ppm level after MS are regenerated. In the 'second optimized configuration' when purifier 1 is saturated (CL1AFTERP1 in Fig. 3) the gas remains dry because the MS $4 \AA$ in purifier 2 still absorbs water. In this configuration, the water concentration inside the gap is easily kept under control for longer periods (about a factor 2 with respect to 'current LHC and the first optimized configurations').

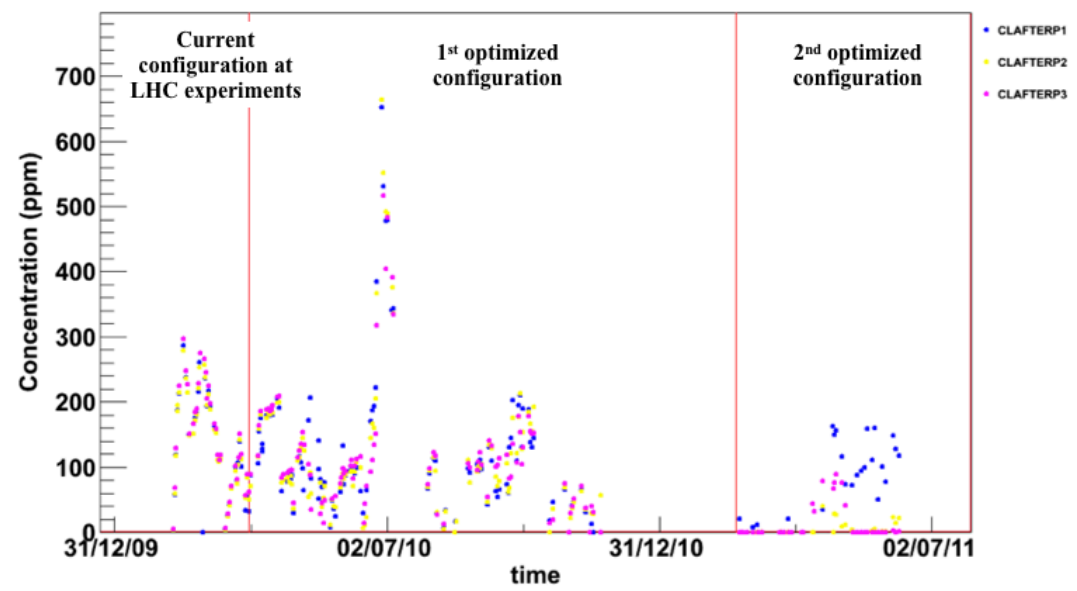

Fig. $3 \mathrm{H}_{2} \mathrm{O}$ concentration after each purifier (dots of different colours) as a function of the time for the three configurations tested at the GIF. 


\subsubsection{Radiation-induced impurities}

Another quality of the second optimized configuration concerns the radiation-induced impurities in the gas. Impurities have been constantly monitored during the long test in order to check their presence and concentrations (in general from $100 \mathrm{ppm}$ to $1000 \mathrm{ppm}$ ). In the final configuration of filters the impurities show with a lower concentration and some even disappear.

The monitor of impurities along time is also useful to understand when the purifiers need to be regenerated: the sudden increase of the pollutants concentration is correlated with the saturation of the purifiers.

\subsubsection{Fresh gas injection}

In the LHC RPC gas systems about $5-10 \%$ of fresh mixture is injected in the circulation loop. At the GIF, a systematic study has been done in order to understand how the impurities concentration changes with a different fraction of fresh mixture injected in the presence of the three purifiers. The attention for this test should be focused on the 'second optimized configuration' test time which can be sub-divided in three periods with different percentages of fresh mixture: 3\% (period A), < 1\% (period B) and 6\% (period C). It has to be considered that at the GIF the dose rate acceleration factor is about 30, so the time period for each test should to be multiplied by this factor to possibly obtain a realistic, equivalent operation time in LHC conditions.

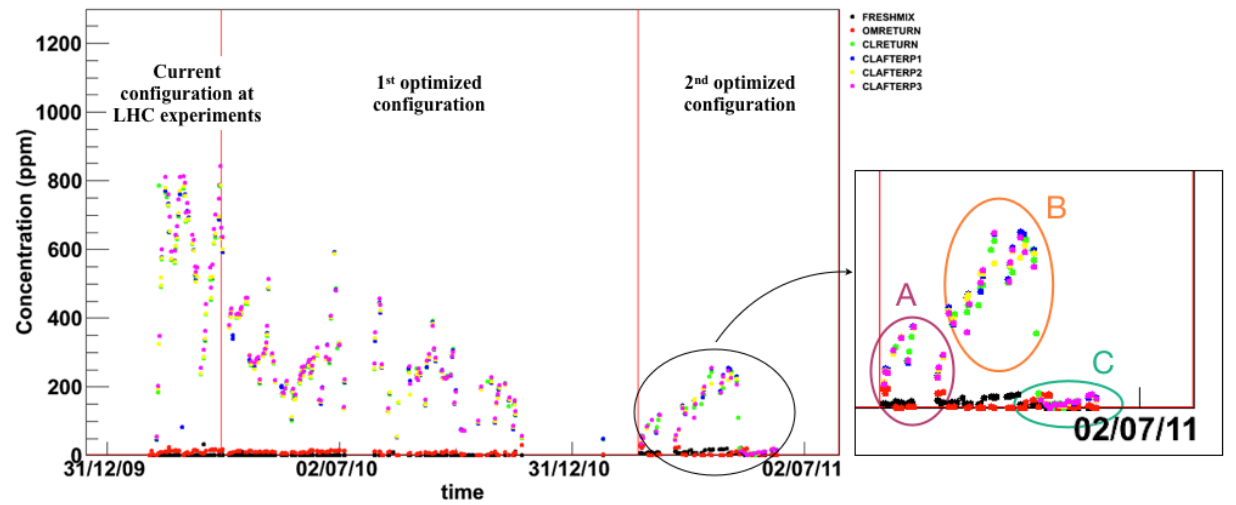

Fig. 4 Concentration of one impurity vs. time during the GIF test. The zoomed region represents the concentration during the test period for the second optimized configuration.

Fig. 4 shows the concentration of one of the detected impurities in the RPC irradiated gas. The pollution concentration is lower for the second optimized configuration with respect to the other periods (where the percentage of fresh mixture injected was always about $7 \%$ ). The impurity concentration gets steadily higher with decreasing fresh gas amount addition. In period $\mathrm{C}$, when $6 \%$ fresh gas is injected in the closed-loop, the impurity concentration remains at the same level of its concentration in chambers operated in open mode, which means that the 
impurity is completely absorbed by the purifiers in the closed-loop. Therefore the injection of the proper amount of fresh gas inside the chamber in closed-loop helps in avoiding an exponential increase of the impurities and it is necessary to safely operate RPC systems.

A test with the closed-loop system operated without purifiers, but just adding $6 \%$ fresh gas in the loop, was also performed. After about 6 days of operation, equivalent to few months at LHC, the impurities concentration increased and some new impurities appeared. Furthermore the water concentration inside the gap could not be kept under control. This means that the purifiers are really needed.

\section{Conclusions}

The 12 large-size RPCs operated at the GIF have been fully exposed to substantial irradiation for about 3 years, corresponding to about 10 years of operation in the LHC systems. During this period several RPC parameters were monitored in order to control the RPC performance and several tests have been performed in order to obtain:

- a systematic and detailed characterization of the effectiveness of several gas cleaningfilters;

- a detection, quantification and identification of the extra-components in the RPC gas mixture created during the harsh irradiation of detectors;

- a complete understanding about performances and limits of the original purifiers configuration in the RPC gas system currently operating at LHC;

- a systematic study of two, new optimized filter configurations that keep the gas of RPC systems operated at high luminosity near the quality of fresh gas mixtures.

The complete understanding of purifiers' filtering capacity allowed the implementation of two optimized configurations. Both configurations were tested at the GIF for a period of one year each. The aim was to find further improvements and to identify the best implementation for the LHC gas systems.

We can conclude that a combination of MS $5 \AA$ and $4 \AA$ together with $\mathrm{Cu}$ R11 is in perfect agreement with the requirements of the large RPC gas systems at the LHC experiments for the following reasons:

- Water is completely filtered, and this allows to re-inject the correct quantity of $\mathrm{H}_{2} \mathrm{O}$ in the gas mixture to maintain constant the Bakelite bulk resistivity.

- One metallic catalyst, Cu R11 is enough to adsorb the O2 present.

- The purifiers cycle duration is increased a factor 2.5 with respect to the current LHC configuration, in particular purifier modules can easily handle an increase of a factor > 1.5 in flow if needed during LHC high luminosity runs.

- This combination removes most gas pollutants created in the irradiated RPCs and accumulated in closed-loop circulation. 
- The performance of heavily irradiated RPCs seems to be unaffected at long-term by the achieved low concentration of impurities: after correction for changes due to environmental conditions (current fluctuations induced by pressure and temperature variations), the currents drawn by the RPCs connected to the optimized closed-loop gas system have been very stable over the entire test period.

\section{References}

[1] M. Capeans et al., Optimization of a closed loop gas system for the operation of Resistive Plate Chambers at the Large Hadron Collider experiments, 2009 IEEE Nuclear Science Symposium Conference Record N11-6, 2009.

[2] S. Altieri et al., The Bakelite for the RPCs of the experiment CMS, Nucl. Instr. and Meth. A 456 (2000) 132.

[3] R. Guida et al., Optimization of a closed-loop gas system for the operation of Resistive Plate Chambers at the Large Hadron Collider experiments, Nucl.Instr. and Meth. A 661 (2012) 214-221.

[4] B. Mandelli et al., Long-term study of optimal gas purifiers for the RPC systems at LHC, CERN PH-EP-Tech-Note-2012-001.

[5] M. Capeans et al., Studies of purification of the Resistive Plate Chamber gas mixture for the Large Hadron Collider experiments, 2008 IEEE Nuclear Science Symposium Conference Record N02-165, 2008.

[6] M. Capeans et al., Long Term Validation of the Optimal Filters Configuration for the Resistive Plate Chambers Gas System at the Large Hadron Collider Experiments, 2011 IEEE Nuclear Science Symposium Conference Record NP5.S-206, 2011. 